\title{
Diversidad de micromamíferos en tres ambientes de la Reserva Nacional Lago Peñuelas, Región de Valparaíso, Chile
}

\section{Diversity of small mammals in three environments of the National Reserve Lago Peñuelas, Región de Valparaíso, Chile}

\author{
Andrés Muñoz-Pedreros ${ }^{1 *}$, Susan Fletcher ${ }^{1}$, José Yáñez ${ }^{2,3}$ \& Pamela Sánchez ${ }^{1}$ \\ ${ }^{1}$ Laboratorio de Ecología Aplicada y Biodiversidad, Escuela de Ciencias Ambientales, Facultad de Recursos Naturales, \\ Universidad Católica de Temuco. \\ ${ }^{2}$ Museo Nacional de Historia Natural. \\ ${ }^{3}$ Centro de Estudios Agrarios y Ambientales. \\ *Email: amunoz@uct.cl
}

\begin{abstract}
RESUMEN
Los ecosistemas mediterráneos de Chile, los únicos en Sudamérica de los cuatro presentes en el mundo, son considerados áreas prioritarias para la conservación, por su alta concentración de especies endémicas y las aceleradas tasas de destrucción del hábitat. Éstos contienen más del 39\% de las especies de mamíferos, $47 \%$ de las especies endémicas y el $65 \%$ de las especies amenazadas del país. Aún así, estos ecosistemas están inadecuadamente representados en el sistema de áreas silvestres protegidas, siendo una de ellas la Reserva Nacional Lago Peñuelas (RNLP) parte de la Reserva de Biosfera La Campana-Peñuelas. La mastofauna de la RNLP esta pobremente documentada. Estudiamos la diversidad $\alpha$ y $\beta$ en el ensamble de micromamíferos presentes en los tres ambientes de la RNLP (bosque esclerófilo, matorral mixto y sabana de Acacia caven). Instalamos grillas de trampas Sherman, recolectamos y analizamos egagrópilas de dos especies de aves rapaces (Tyto alba y Bubo magellanicus) y registramos huellas, signos y observación directa en las cuatro estaciones del año 2001. Determinamos la riqueza de especies (S), abundancia relativa, diversidad $\alpha$ considerando su riqueza y estructura (índices de Shannon y Wiener y de Pielou), diversidad $\beta$ (índice de Bray-Curtis) y comparamos la diversidad encontrada con lo documentado en la misma latitud de este a oeste. Registramos un total de 16 especies: Thylamys elegans, Oligoryzomys longicaudatus, Abrothrix longipilis, A. olivaceus, Chelemys megalonyx, Phyllotis darwini, Myocastor coypus, Octodon degus, O. lunatus, Spalacopus cyanus, Abrocoma bennetti, Rattus norvegicus, $R$. rattus, Mus musculus, Lepus capensis y Oryctolagus cuniculus. El bosque esclerófilo costero fue el más diverso con distribución homogénea de sus especies, seguido del matorral mixto y finalmente la sabana de $A$. caven. Por otro lado el bosque esclerófilo es similar al matorral mixto y ambos a su vez muy disímiles de la sabana de $A$. caven. La diversidad registrada en el área de estudio es concordante con la correspondiente a otras áreas mediterráneas de Chile. Se discute la situación de estos ecosistemas y se contrasta la diversidad documentada para seis localidades a la misma latitud.
\end{abstract}

Palabras clave: Biodiversidad, mamíferos, roedores, Chile central.

\begin{abstract}
Chilean Mediterranean ecosystems, the only of this type present in South America of the four present in the world, are considered priority areas for conservation due to their high concentration of endemic species that have experienced accelerated rates of habitat destruction. They contain more than $39 \%$ of the mammal species, $47 \%$ of its endemic species, and $65 \%$ of the threatened species of Chile. Yet, these ecosystems are poorly represented in the system of protected areas, one of which is the Reserva Nacional Lago Peñuelas (RNLP) that is part of the Biosphere Reserve La Campana-Peñuelas, but whose mammal fauna is poorly documented. We studied both $\alpha$ and $\beta$ diversity of the mammal assemblage in all three environments present at the RNLP (sclerophyllous forest, mixed shrub, and savanna of Acacia caven). Sherman traps grids were installed, pellets of two raptors (Tyto alba and Bubo magellanicus) were analyzed, tracks and signs were recorded, and direct observations were performed in the four seasons of the year 2001. We determined species richness (S), relative abundance, $\alpha$ diversity - considering its richness and structure (Shannon-Wiener and Pielou indexes)-, $\beta$ diversity (BrayCurtis index), and compared the diversity found with that documented for the same latitude from east to west. We recorded a total of 16 species: Thylamys elegans, Oligoryzomys longicaudatus, Abrothrix longipilis, A. olivaceus, Chelemys megalonyx, Phyllotis darwini, Myocastor coypus, Octodon degus, O. lunatus, Spalacopus cyanus, Abrocoma bennetti, Rattus norvegicus, R. rattus, Mus musculus, Lepus capensis, and Oryctolagus cuniculus. The coastal sclerophyllous forest was the most diverse with a species homogeneous distribution, followed by the mixed shrub, and finally the $A$. caven
\end{abstract}


savanna. Also, the sclerophyllous forest is similar to the mixed scrub; in turn, both are very dissimilar to the savanna of A. caven. The diversity recorded in the study area is consistent with that of other areas of the Mediterranean areas. We discuss the status of these ecosystems and the diversity is compared to six documented locations at the same latitude.

KeYwords: Biodiversity, mammals, rodents, Central Chile.

\section{INTRODUCCIÓN}

La biodiversidad o diversidad biológica es la suma total de toda la variación biótica desde el nivel de genes al de ecosistemas (véase Purvis \& Raven (2000). La diversidad biológica de Chile comparada con países tropicales es bastante menor, sin embargo posee un alto nivel de endemismo atribuible, principalmente, a factores biogeográficos (Naranjo 1993). Por ejemplo, de las 369 especies de mamíferos que existen en Ecuador sólo 30 son endémicas $(8,1 \%)$ (Tirira 1999), mientras que en Chile de las 157 especies de mamíferos registradas (Yáñez \& MuñozPedreros 2009) 37 son endémicas (23,6\%) (Simonetti et al. 1995). Este marcado endemismo que presenta Chile se hace más evidente en su porción central mediterránea, constituyendo el área geográfica nacional con mayor endemismo y cuyas características han determinado que se considere a la región de Chile central como uno de los 25 puntos prioritarios (hotspots) de biodiversidad a nivel global (Myers et al. 2000).

Es más, los ecosistemas mediterráneos son considerados como puntos prioritarios para la conservación a nivel mundial, ya que son áreas de alta concentración de especies endémicas, las cuales han experimentado aceleradas tasas de destrucción del hábitat (cf. Medail \& Quezel 1997, Myers et al. 2000, Ceballos \& Ehrlich 2006). Por otro lado esta ecorregión mediterránea es la única en Sudamérica (sensu Dinerstein et al. 1995) y destaca por sus altos niveles de endemismos, con muchos géneros de plantas altamente diversificados y una alta riqueza de especies (Arroyo 1999), albergando más del 39\% de las especies de mamíferos chilenos, el $47 \%$ de las especies endémicas chilenas y el 65\% de las especies amenazadas (Simonetti 1999).

Pese a la extensa cobertura del Sistema Nacional de Áreas Silvestres Protegidas del Estado de Chile (SNASPE), algunas regiones ecológicas de Chile están inadecuadamente representadas (Mardones 1996), como es el caso de los ecosistemas mediterráneos, que cubren un $16 \%$ del territorio y que se despliega desde el norte de la Región de Coquimbo hasta el sur de la Región del Maule (Di Castri 1973). Sin embargo sólo el 0,9\% de estos ecosistemas están representados en el total del SNASPE (Mardones 1996), estando subrepresentados (Mella \& Simonetti 1994) y con cambios antropogénicos profundos en el paisaje (Greuter 1995). Una de las áreas silvestres protegidas que mantiene ecosistemas de tipo mediterráneo en Chile, es la Reserva Nacional Lago Peñuelas (RNLP) ubicada en la Región de Valparaíso, que junto con el Parque Nacional La Campana (PNLC), es Reserva de Biosfera (La Campana-Peñuelas de 17905 ha). En la RNLP existe un bosque esclerófilo costero de clima mediterráneo que se encuentra en una crítica situación a nivel nacional y posee pocos estudios de la diversidad de micromamíferos nativos.

En el análisis de la diversidad a nivel de especies, Whittaker (1960) la separó en diversidad alfa $(\alpha)$, que corresponde al número de especies de una comunidad homogénea determinada, la diversidad beta $(\beta)$, entendida como el grado de cambio o reemplazo en la composición de especies entre diferentes comunidades presentes en un paisaje y la diversidad gamma $(\gamma)$, que es la riqueza de especies del conjunto de comunidades que integran un paisaje, resultado de la combinación de la diversidad alfa y beta (Whittaker 1972, Whittaker et al. 2001, Moreno 2001). El recambio de especies $(\beta)$ es el componente menos estudiado de la biodiversidad, pese a sus claras implicancias en la conservación, ya que es un elemento vital para comprender y comparar la diversidad local y regional (Cornell \& Lawton 1992, Ricklefs \& Schluter 1993, Scott et al. 1999, Gaston \& Blackburn 2000).

La diversidad $\beta$ es de alta importancia ya que, por ejemplo, dos regiones de alta diversidad $(\gamma)$ podrían contener similar número de especies, pero tener diferentes diversidades $\alpha$ y $\beta$. Es decir podríamos explicar la alta diversidad $\gamma$, en un caso, porque sus localidades tienen alta diversidad $\alpha$ compartiendo las mismas especies (baja diversidad $\beta$ ), en el otro caso porque existe un fuerte recambio de especie (alta diversidad $\beta$ ), aunque la diversidad de las localidades $(\alpha)$ no sea alta. De esta forma para conservar las especies, en el primer caso, se requerirían pocas áreas protegidas, pero en el segundo caso se requerirían de varias (véase Rodríguez et al. 2003).

El objetivo de este trabajo es caracterizar la diversidad de micromamíferos $(\alpha$ y $\beta$ ) en la RNLP y analizar su representación de la subregión del bosque esclerófilo costero (sensu Gajardo 1994). Esta información es útil en el diseño de estrategias para la conservación de micromamíferos a diferentes escalas, especialmente en la zonificación de la reserva contenida en su plan de manejo. 


\section{MATERIALES Y METODOS}

AREA DE ESTUdio

El estudio se realizó en la Reserva Nacional Lago Peñuelas (3307’'S; $\left.71^{\circ} 24^{\prime} \mathrm{O}\right)$ ubicada en la Región de Valparaíso, Chile. El clima es del tipo templado cálido con humedad suficiente, caracterizada por periodos de lluvia regulares durante el invierno y una estación seca bien marcada, que puede extenderse entre seis y ocho meses (codificados como Cfa, Csb sensu Köppen 1948). La precipitación es de alrededor de $382,3 \mathrm{~mm} / \mathrm{año}$, el $80 \%$ de la cual se registra entre mayo y agosto. La temperatura media es de $14,9^{\circ} \mathrm{C}$, con una máxima media de $22,2^{\circ}$ y una mínima media de $9,8^{\circ} \mathrm{C}$ (Estación Peñablanca en Di Castri \& Hajek 1976). La RNLP posee una superficie de 9260 ha y el área de estudio comprendió las 2473 ha con cubierta vegetal natural, estando la superficie restante cubierta por vegetación exótica, dominada por plantaciones de pino insigne (Pinus radiata D. Don) y eucalipto (Eucalyptus globulus Labill.). Otra superficie corresponde al espejo de agua del lago Peñuelas. En el área de estudio se seleccionaron tres ambientes representativos: sabana de Acacia caven (Molina) Molina, bosque esclerófilo costero y matorral mixto de A. caven y Baccharis linearis (Ruiz et Pav.) Pers. En cada uno de éstos se realizaron los estudios de microhábitat y los registros estacionales de micromamíferos más adelante descritos.

\section{Metodología}

Para la caracterización de los ambientes se empleó la información de un estudio fitosociológico y florístico sincrónico (Hauenstein et al. 2009) en donde se determinó el valor de importancia de las especies según Wikum \& Shanholtzer (1978), se describieron las formas de vida según Ellenberg \& Mueller-Dombois (1966) y el origen fitogeográfico según Marticorena \& Quezada (1985) y Matthei (1995). También se determinó el grado de perturbación antrópica de los tres ambientes sobre la base de lo propuesto por Hauenstein et al. (1988), que consideran el origen fitogeográfico, es decir, la relación entre las especies nativas e introducidas.

La información sobre las poblaciones de micromamíferos se obtuvo por medio de la instalación de una grilla de $60 \mathrm{x}$ 60 m (sensu Muñoz-Pedreros \& Yáñez 2009) en cada uno de los ambientes caracterizados, desplegándose en total 108 trampas del tipo Sherman y 15 trampas Tomahawk, por tres noches durante las cuatro estaciones de 2001, empleándose como cebo avena cruda machacada. Así en cada grilla se desplegaron los mismos tipos y números de trampas, sometiéndose los animales capturados en los retículos a procedimiento de rutina (sensu DeBlase \& Martin 1974). La nomenclatura siguió a Yáñez \& Muñoz-Pedreros (2009), los animales fueron marcados usando pintura de colores diferentes en cada estación. Para la manipulación de los animales se usaron equipos de bioseguridad aprobados por el Ministerio de Salud de Chile como precaución para trabajar con animales seropositivos al virus Hanta. Con el fin de complementar la información del inventario de micromamíferos se empleó información de un trabajo sincrónico sobre ecología trófica de aves rapaces nocturnas del lugar (Muñoz-Pedreros et al. 2010 información no publicada), en que se recolectaron egagrópilas de las estrigiformes Tyto alba Scopoli y Bubo magellanicus Gmelin obtenidas en el sector la Pérgola y en las cercanías de la Vega del Álamo. A éstas se agregaron los registros de muestras de T. alba analizadas por Maldonado (1986). Las egagrópilas fueron estudiadas siguiendo a Reise (1973), Pearson (1995) y Muñoz-Pedreros \& Rau (2004).

Para la asociación de micromamíferos se determinó: (a) La riqueza de especies (S) entendida como el número de especies en una muestra. (b) La abundancia relativa (AB\%), entendida como la fracción porcentual del total de animales (sensu Krebs 1985), lo que permitió identificar las especies de baja representatividad (poco abundantes). (c) La diversidad $\alpha$ (intra-ambiente), considerando la riqueza específica y la estructura. Esta última se determinó según el índice de diversidad de Shannon y Wiener, que cuantifica la diversidad total de una muestra, siendo influida por dos componentes fundamentales: la riqueza y la equidad. Así considera el valor de importancia de cada especie y expresa la uniformidad de los valores de importancia a través de todas las especies de la muestra. La fórmula para esta función es: $\mathrm{H}^{\prime}=-\Sigma\left(p_{i} \times \log _{2}\right.$ $\left.p_{i}\right)$, donde $p_{i}$ es la proporción del número total de individuos de la muestra que corresponde a la especie, cuyos valores se despliegan entre cero cuando existe sólo una especie, y el máximo (H'máx) que corresponde al $\log _{2} \mathrm{~S}$. Además, se calculó el índice de equidad de Pielou $(\mathrm{J})$ según la ecuación: $\mathrm{J}=\mathrm{H}^{\prime} / \mathrm{H}^{\prime}$ máx. Este índice cuantifica la contribución de la equidad a la diversidad total observada. Sus valores fluctúan entre 0 (mínima heterogeneidad) y 1 (máxima heterogeneidad, es decir, las especies son igualmente abundantes) (Magurran 1998, Moreno 2001). Para probar la hipótesis nula que la diversidad $\mathrm{H}^{\prime}$ de los tres ambientes son iguales se siguió el procedimiento de Hutcheson (1970) descrito en Zar (1996), consistente en un test de $t$ calculando el índice de diversidad ponderado $\left(\mathrm{Hp}=(N \log N)-\left(\sum f_{i} \log f_{i}\right) / N\right)$, incluido el cálculo de su varianza para cada ambiente según $\mathrm{S}_{\mathrm{H}}{ }^{2}=\left[\sum f_{i} \log ^{2} f_{i}\right.$ $\left.-\left(\sum f_{i} \log f_{i}\right)^{2} / N\right] / N^{2}$. (d) La diversidad $\beta$ (entre ambientes) se representó por el recambio de especies mediante un cluster o dendrograma de similitud/disimilitud (entre los ambientes) basado en el índice de Bray \& Curtis (1957), utilizando el programa BioDiversity Professional (McAleece 1998). (e) Realizamos registros de huellas, signos y observación directa en la red de caminos y senderos de la reserva para registrar especies no capturables con trampas Sherman (e.g., lagomorfos y coipos). (f) Finalmente contrastamos la 
diversidad encontrada con la diversidad de micromamíferos documentada en la misma latitud.

\section{RESULTADOS}

CARACTERIZACIÓN VEGETACIONAL DE LOS AMBIENTES

El primer ambiente correspondió a una sabana de $A$. caven en la que la especie dominante, el espino, se encuentra rodeado de extensos pastizales. En esta formación vegetal predominaron sólo dos especies leñosas: $A$. caven con coberturas promedio de $30 \%$ y Maytenus boaria Molina cuyas coberturas fueron inferiores al 10\%. El estrato herbáceo alcanzó una cobertura del 90\%, representado por especies como Agrostis capillaris L., Leontodon saxatilis Lam. y Poa annua L., existiendo un claro predominio de gramíneas forrajeras. Respecto a la flora, se registraron 20 especies, de las cuales el 55\% correspondió a especies introducidas, existiendo, por lo tanto, un grado relativamente alto de intervención antrópica en el lugar (sensu Hauenstein et al. 1988). El espectro biológico de este sitio mostró claramente que las formas de vida predominantes correspondieron a las terófitas, hemicriptófitas y criptófitas. En menor porcentaje estaban representadas las fanerófitas con especies como $M$. boaria y Quillaja saponaria Molina, y las nanofanerófitas que estaban representadas por una sola especie (A. caven). Este espectro biológico concuerda claramente con las condiciones de sequía del lugar, especialmente en la época estival, ya que los terófitos (plantas herbáceas de ciclos de vida cortos, anuales o bienales) y los criptófitos (geófitos o plantas con órganos subterráneos perdurantes) representan muy bien este tipo de clima y son buenos indicadores ambientales. Por su parte, la abundancia de hemicriptófitos indica más bien intervención humana, ya que esta forma de vida acompaña al ser humano y corresponde a plantas que son capaces de soportar el pisoteo y ramoneo de los animales domésticos (Cabrera \& Willink 1973, Ramírez 1988, Grigera et al. 1996).

El segundo ambiente correspondió a un bosque esclerófilo en donde la cobertura promedio del estrato arbóreo fue de $70 \%$, la de arbustos $35 \%$ y la herbácea de $25 \%$. Destacan en el estrato arbóreo especies como Peumus boldus Molina, Q. saponaria y Cryptocarya alba (Molina) Looser, en el arbustivo Retanilla trinervia (Guillies et Hook.) Hook. et Arn., Schinus latifolia (Gillies ex Lindl.) Engler y Azara dentata Ruiz et Pav. y en el estrato herbáceo, Alonsoa meridionalis (L.f.) Kuntze, Conyza sumatrensis (Retz.) E.Walker var. floribunda (Kunth) J.B. Marshall, Anagallis arvensis L. y Marrubium vulgare L. A esta comunidad se le conoce con el nombre científico de Peumo-Cryptocaryetum albae (Oberdorfer 1960). Es posible encontrar algunas variantes de esta comunidad con especies asociadas al matorral esclerófilo. Se registraron
31 especies de flora, de las cuales el $22,5 \%$ son especies introducidas. Respecto a sus formas de vida, existió un predominio de fanerófitas que requieren de condiciones de pluviometría mayores que las otras formas de vida registradas. El espectro biológico está en concordancia con las características climáticas del área, porque las especies presentaron adaptaciones características de las esclerófilas. Las terófitas y hemicriptófitas presentaron, en las evaluaciones, importantes valores de abundancia.

Finalmente, el tercer ambiente correspondió a un matorral mixto de $A$. caven y $B$. linearis. Las especies leñosas dominantes fueron $A$. caven, $B$. linearis, M. boaria, $Q$. saponaria y $S$. latifolius; con menor cobertura se registraron P. boldus, Lithraea caustica (Molina) Hook. et Arn., Escallonia pulverulenta (Ruiz et Pav.) Pers. y la trepadora Muehlenbeckia hastulata (Sm.) I.M. Johnst. El estrato herbáceo, cuya cobertura promedio fue de $90 \%$, estuvo representado por: L. saxatilis, A. capillaris, Avena barbata Pott ex Link, Festuca sp., P. annua, Briza minor L. y Bromus hordeaceus L. Se registraron 36 especies de flora, de las cuales el 30,5\% correspondió a especies introducidas. En sus formas de vida, aquí son importantes las fanerófitas de características esclerófilas y las terófitas, la presencia importante de hemicriptófitas indica alteración antrópica del lugar.

\section{REGISTROS DE MICROMAMÍFEROS}

Se realizaron 181 capturas de micromamíferos en 1476 trampas noches (12,9\% de éxito), capturándose 10 especies: llaca elegante, Thylamys elegans (Waterhouse, 1839); ratón de los espinos, Oligoryzomys longicaudatus (Bennett, 1832); laucha de pelo largo, Abrothrix longipilis (Waterhouse, 1837); laucha olivácea, Abrothrix olivaceus (Waterhouse, 1837); lauchón orejudo de Darwin, Phyllotis darwini (Waterhouse, 1837); degú de las pircas, Octodon degus (Molina, 1782); degú costino, Octodon lunatus Osgood, 1943; rata chinchilla de Bennett, Abrocoma bennetti (Waterhouse, 1837); guarén, Rattus norvegicus (Berkenhout, 1769); y rata negra, Rattus rattus (Linnaeus, 1758).

Del estudio de las egagrópilas de T. alba y B. magellanicus (469 muestras, incluidas aquéllas de Maldonado 1986) se registró un total de 646 presas de vertebrados, de las cuales el 90,3\% correspondió a micromamíferos. En las egagrópilas se identificaron una especie de marsupial (T. elegans), 12 roedores (O. longicaudatus, A. longipilis. A. olivaceus, la rata topo del matorral Chelemys megalonyx (Waterhouse, 1844), P. darwini, O.degus, O. lunatus, el coruro Spalacopus cyanus (Molina, 1782), A. bennetti, $R$. norvegicus, $R$. rattus y la laucha Mus musculus Linnaeus, 1758) y el lagomorfo Oryctolagus cuniculus (Linnaeus, 1758). Nótese que tres roedores (i.e., C. megalonyx, S. cyanus y M. musculus) no fueron capturados con trampas Sherman y Tomahawk. 
Se registraron, huellas y madrigueras de coipo, Myocastor coypus (Molina, 1782) en el sector este del lago y tranque de alimentación (febrero de 2001); luego fueron avistados cuatro ejemplares, dos adultos y dos crías, en el sector la Estancia (junio de 2001). En recorridos diurnos y nocturnos se registraron ejemplares de $O$. cuniculus y de la liebre Lepus capensis Linnaeus, 1758. Es así que la RNLP posee registros de 16 especies de micromamíferos, incluyendo cinco especies introducidas.

RIQUEZA Y ABUNDANCIA DE ESPECIES DE MICROMAMÍFEROS De las especies registradas mediante trampeos el ambiente con mayor riqueza fue el bosque esclerófilo con 10 especies de micromamíferos. En el matorral mixto se registraron nueve especies y finalmente en la sabana de $A$. caven sólo se registraron dos especies. El ambiente con mayor abundancia de micromamíferos correspondió al matorral mixto $(54,7 \%)$ seguido del bosque esclerófilo $(36,5 \%)$ y por último la sabana de $A$. caven $(8,8 \%)$.
En el matorral mixto la especie más abundante fue $A$. olivaceus (64\%) seguida de $O$. degus $(15,1 \%)$. En el bosque esclerófilo $R$. norvegicus, una especie exótica, destacó con una abundancia del 21,2 \%, le siguió la especie nativa $O$. longicaudatus con un $18,1 \%$. En la sabana de $A$. caven, donde sólo dos especies fueron capturadas, $A$. olivaceus presentó una alta abundancia (94\%) comparado con $O$. longicaudatus $(6,2 \%)$. En toda el área de estudio las especies más abundantes fueron $\mathrm{A}$. olivaceus (47\%), O. degus $(12,7 \%)$ у O. longicaudatus $(11,6 \%)$.

La abundancia de micromamíferos registrada en cada uno de los ambientes del área de estudio presentó variaciones estacionales. Así, en el ambiente de sabana de $A$. caven se registró la mayor abundancia en otoño $(14,2 \%)$ y en verano la mínima (4,9\%). En el bosque esclerófilo la abundancia varió entre $45,7 \%$ en invierno y $24,5 \%$ en verano. En el matorral mixto durante el verano se registró el mayor valor de abundancia $(70,4 \%)$ y en otoño-invierno el menor

Tabla 1. Capturas de micromamíferos en tres ambientes de la RN Lago Peñuelas, Chile central. Frecuencia de individuos (F), Abundancia relativa (AB\%), Riqueza de especies (S), Número de individuos (N), Diversidad (H'), Diversidad máxima (H'max), Índice de equiparabilidad (E), Índice de Simpson (D) y Diversidad máxima de Simpson (D máx.).

TABLE 1. Captures of small mammals in three environments of RN Lago Peñuelas, central Chile. Frequency of individuals (F), Relative abundance (AB\%), Species richness (S), Number of individuals (N), Diversity (H'), Maximum diversity (H' max), the equitability index (E), Simpson's index (D) and Simpson's maximum diversity (D max.)

\begin{tabular}{|c|c|c|c|c|c|c|c|c|c|}
\hline \multirow[t]{2}{*}{ Especies } & \multirow[t]{2}{*}{ Nombre común } & \multicolumn{2}{|c|}{$\begin{array}{l}\text { Sabana de } \\
\text { A. caven }\end{array}$} & \multicolumn{2}{|c|}{$\begin{array}{l}\text { Bosque } \\
\text { esclerófilo }\end{array}$} & \multicolumn{2}{|c|}{$\begin{array}{c}\text { Matorral } \\
\text { mixto }\end{array}$} & \multicolumn{2}{|c|}{ Total } \\
\hline & & $\mathrm{F}$ & $\mathrm{AB} \%$ & $\mathrm{~F}$ & $\mathrm{AB} \%$ & $\mathrm{~F}$ & $\mathrm{AB} \%$ & $\mathrm{~F}$ & $\mathrm{AB} \%$ \\
\hline Thylamys elegans & llaca elegante & - & - & 4 & 6,1 & 4 & 4,0 & 8 & 4,4 \\
\hline Oligoryzomys longicaudatus & ratón de los espinos & 1 & 6,2 & 12 & 18,1 & 8 & 8,1 & 21 & 11,6 \\
\hline Abrothrix longipilis & laucha de pelo largo & - & - & 7 & 10,6 & 2 & 2,0 & 9 & 5,0 \\
\hline Abrothrix olivaceus & laucha olivácea & 15 & 94,0 & 7 & 10,6 & 63 & 64,0 & 85 & 47,0 \\
\hline Phyllotis darwini & lauchón orejudo de Darwin & - & - & 1 & 1,5 & - & - & 1 & 0,5 \\
\hline Octodon degus & degú de las pircas & - & - & 8 & 12,1 & 15 & 15,1 & 23 & 12,7 \\
\hline Octodon lunatus & degú costino & - & - & 1 & 1,5 & 1 & 1,0 & 2 & 1,1 \\
\hline Abrocoma bennetti & rata chinchilla de Bennett & - & - & 9 & 13,6 & 3 & 3,0 & 12 & 6,6 \\
\hline Rattus norvegicus & guarén & - & - & 14 & 21,2 & 1 & 1,0 & 15 & 8,2 \\
\hline Rattus rattus & rata negra & - & - & 3 & 4,5 & 2 & 2,0 & 5 & 2,7 \\
\hline Total & & 16 & 8,8 & 66 & 36,5 & 99 & 54,7 & 181 & 100 \\
\hline $\mathrm{S}$ & & & 2 & & 10 & & 9 & & 10 \\
\hline $\mathrm{N}$ & & & 16 & & 66 & & 99 & & 181 \\
\hline $\mathrm{H}^{\prime}$ & & & 0,34 & & 3,00 & & 1,82 & & 2,47 \\
\hline H'máx & & & 1,00 & & 3,32 & & 3,17 & & 3,32 \\
\hline $\mathrm{E}$ & & & 0,33 & & 0,90 & & 0,57 & & 0,74 \\
\hline
\end{tabular}


$(45,7 \%)$. En el área de estudio en su conjunto la estación con mayor abundancia fue verano $(33,7 \%)$, seguida de primavera $(27,6 \%)$ y por último otoño e invierno (19,3\%).

\section{DIVERSIDAD DE MICROMAMÍFEROS}

En el ambiente de bosque esclerófilo, el índice de diversidad $\left(\mathrm{H}^{\prime}\right)$ fue de 3,00 bits con un $\mathrm{H}^{\prime}$ máx $=3,32$ bits. En el ambiente de matorral mixto el $\mathrm{H}^{\prime}$ fue de 1,82 bits con un $\mathrm{H}^{\prime}$ máx $=3,17$ bits. Finalmente, en el ambiente de sabana de $A$. caven el $\mathrm{H}^{\prime}$ fue de 0,34 con un $\mathrm{H}^{\prime}$ máx $=1$ (ver Tabla 1). En resumen, el bosque esclerófilo costero fue el más diverso seguido del matorral mixto y finalmente la sabana de $A$. caven. Respecto al índice de equidad de Pielou (E), los valores muestran que en el ambiente de sabana de $A$. caven existe una desigualdad en las abundancias de las especies registradas $(\mathrm{E}=0,33)$ debido a que una de las especies (A. olivaceus) presentó un valor de abundancia mucho mayor que la otra $(O$. longicaudatus). Mientras que lo opuesto se presenta en el caso del bosque esclerófilo donde el valor de $\mathrm{E}$ indica una distribución homogénea $(E=0,90)$ entre las abundancias de las especies registradas (Tabla 1).

Existen diferencias significativas en la diversidad ( $\left.\mathrm{H}^{\prime}\right)$ de los tres ambientes. Entre la sabana de $A$. caven y bosque esclerófilo $\left(t_{0,05(2) 22,8}=2,74\right)$, entre la sabana de $A$. caven y el matorral mixto $\left(t_{0,05(2) 37,6}=2,02\right)$ y entre el bosque esclerófilo y el matorral mixto $\left(t_{0,05(2) 150,9}=1,96\right)$. Los valores de $t$ obtenidos son mayores que los valores de $t$ en tablas (133,5 para $A$. caven y bosque esclerófilo, 55,75 $A$. caven y matorral mixto y 88,75 para bosque esclerófilo y matorral mixto), por lo que se rechaza la hipótesis nula y se concluye que la diversidad de micromamíferos no es igual entre los tres ambientes estudiados.

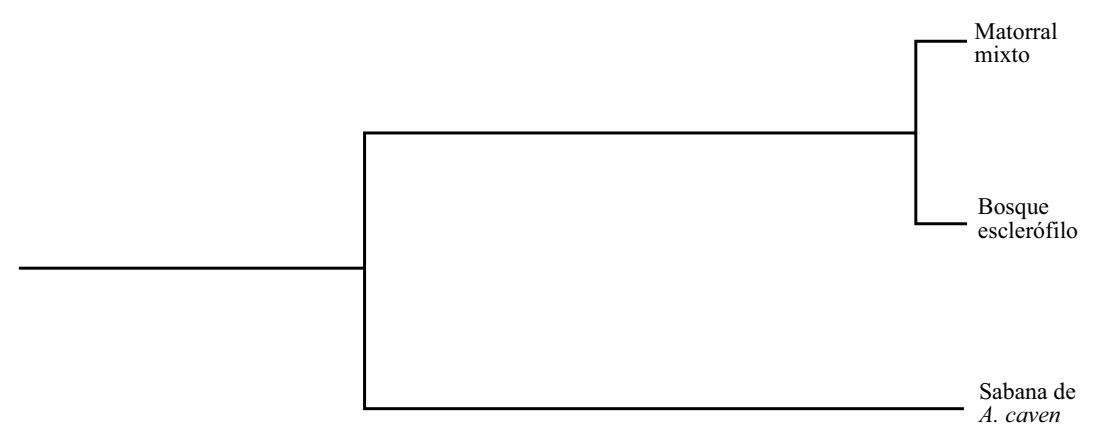

$0 \%$ similitud

50

100

FIgURA 1. Relaciones de similitud del ensamble de micromamíferos en tres ambientes de la Reserva Nacional Lago Peñuelas, Chile central.

FIGURE 1. Similarity relationships of small mammal assembly in three environments of Lago Peñuelas National Reserve, Central Chile.

Para la diversidad $\beta$ se presenta el árbol de agrupamiento resultante del índice de similitud (Fig. 1), en donde se pueden distinguir los tres ambientes. El bosque esclerófilo es similar al matorral mixto $(94,75 \%)$ y ambos a su vez muy disímiles a la sabana de $A$. caven (sólo $33,33 \%$ con el bosque esclerófilo y $36,36 \%$ con el matorral mixto). La baja similitud que presenta la sabana de $A$. caven respecto de los otros ambientes se debe al bajo número de especies registradas. De esta manera, el bosque esclerófilo y el matorral mixto son los ambientes que representan la diversidad más alta de micromamíferos de la RNLP.

En la región en que está ubicada la RNLP, la región del matorral y del bosque esclerófilo (subregión del bosque esclerófilo costero), hay registros de 13 especies nativas de micromamíferos: $T$. elegans, $O$. longicaudatus, A. longipilis, A. olivaceus, C. megalonyx, P. darwini, Lagidium viscacia (Molina, 1782), Chinchilla lanigera (Molina, 1782), M. coypus, O. degus, O. lunatus, S. cyanus, $A$. bennetti; y cinco especies introducidas: $R$. norvegicus, $R$. rattus, M. musculus, L. capensis y O. cuniculus.

\section{DISCUSIÓN}

Respectoalariqueza de especies nativas demicromamíferos, en la Reserva Nacional Lago Peñuelas (RNLP) se registraron 9 especies de las 13 especies esperables para 
la región del matorral y del bosque esclerófilo (sensu Gajardo 1994). No se capturaron las siguientes especies: (a) Chinchilla lanigera que se distribuye más al norte con una población bien documentada en Aucó ( $\left.31^{\circ} 29^{\prime} \mathrm{S}, 71^{\circ} 06^{\prime} \mathrm{O}\right)$, al norte de Illapel en matorrales xerófitos con presencia marginal de bosque esclerófilo costero (Muñoz-Pedreros \& Gil 2009); y (b) Lagidium viscacia que Pine et al. (1979) documentan para las zonas altas del Parque Nacional La Campana (PNLC).

En la Tabla 2 se muestra la diversidad, como riqueza de especies, de micromamíferos en seis localidades en la misma latitud $\left(33^{\circ} \mathrm{S}\right)$. La riqueza de especies nativas de la RNLP es levemente más baja (una especie menos) que la documentada para el PN La Campana, aunque esta área protegida la triplica en superficie, la duplica en número de formaciones vegetacionales y rangos altitudinales (esto último explica la presencia de $L$. viscacia en el PN La Campana). Respecto a lo documentado para la RN Río Clarillo, la diversidad en la RNLP es mayor (una especie más) pese a que la superficie de Río Clarillo es cinco veces mayor y el rango altitudinal va de los 870 a los $3050 \mathrm{msm}$ y posee al menos tres grandes formaciones vegetacionales; además no es esperable en la RNLP por distribución y hábitat la presencia de L. viscacia y Loxodontomys micropus (Waterhouse, 1837). Respecto a esta última especie (documentada por Díaz et al. 2002) es altamente probable que se trate de Loxodontomys pikumche Spotorno et al. 1998, sin embargo ambas especies no son esperables en la RNLP. Las localidades de los alrededores de Santiago presentan una diversidad menor, aun cuando en estos estudios los pequeños mamíferos no capturables en trampas del tipo Sherman no eran aparentemente especies objetivo para los autores (e.g., Fulk 1975, Glanz 1977a,b, Jaksic \& Yáñez 1978, Iriarte et al. 1989); es necesario notar que Jaksic (1997) indica que fueron observados $S$. cyanus y O. cuniculus en la localidad de San Carlos de Apoquindo (Tabla 2).

Existen especies, aunque registradas en una localidad, cuyas poblaciones parecen estar no sólo en retroceso sino que en proceso de extinción local. Por ejemplo, la especie Chelemys megalonyx (distribuida en la cordillera costera desde la provincia del Elqui a la de Cautín) registrada en 1986 en la RNLP en base un único ejemplar de un total de 156 presas provenientes de 70 egagrópilas de T. alba, no fue colectada en este estudio (646 presas de 413 egagrópilas y 1476 trampas/ noches). Nótese que C. megalonyx es una especie cavícola, lo que sugiere que es muy específica para seleccionar su hábitat (Quintana 2009), lo que a su vez propicia que tenga una distribución muy fragmentada. Algo similar ha ocurrido en el PNLC donde Simonetti \& Walkowiak (1979) y Zunino \& Arcos (1989) no registraron a C. megalonyx sobre 201 especímenes de pequeños mamíferos recuperados de 159 egagrópilas; luego Zunino (1990) capturó dos ejemplares que quizá pertenecían a poblaciones muy reducidas y confinadas a quebradas con vegetación densa y cursos de agua (véase Osgood 1943, Mann 1978). Similar es el caso de Myocastor coypus, especie ampliamente distribuida en América del Sur, desde el nivel del mar a los 1100 msm (Muñoz-Pedreros \& Gil 2009). En la RNLP su presencia está circunscrita a pocos lugares con reducidas poblaciones; de forma similar Zunino (1990) considera a esta especie prácticamente desaparecida del PNLC con una sola observación, fuera del área protegida, en el sector de Ocoa.

El matorral mixto, aunque tiene una especie menos $(P$. darwini) que el bosque esclerófilo, presenta una mayor abundancia, pese a que mostró ser un ambiente con alto grado de perturbación antrópica, con un gran porcentaje de especies vegetales exóticas y con un espectro biológico donde predominan las fanerófitas de características esclerófilas, las terófitas y hemicriptófitas (Hauenstein et al. 2009). Esta mayor abundancia en los matorrales también ha sido documentada por Iriarte et al. (1989) en San Carlos de Apoquindo (Región Metropolitana), quienes encontraron que el patrón de uso del hábitat está asociado a la cobertura arbustiva. Algo similar encontraron MuñozPedreros et al. (1990) en un matorral esclerófilo degradado en Burca (provincia de Concepción) indicando que los micromamíferos sólo usan el suelo cuando está cubierto de ramas, lo que revela la importancia de la depredación en la selección del microhábitat, así como la provisión de alimento. Muñoz-Pedreros (1992), en un estudio de ensambles de micromamíferos en un trayecto latitudinal de Chile, también mostró que las densidades de pequeños mamíferos en matorrales son consistentemente mayores que en los bosques, a pesar que la riqueza de especies es mayor en los bosques.

La otra formación vegetal característica de la RNLP, y en general de la zona central de Chile, es la sabana de Acacia caven, con dos estratos, arbustivo y herbáceo anual, por ello se puede definir como una sabana de espino y no estepa. En el área de estudio se observó la dominancia casi absoluta de $A$. caven con coberturas muy ralas y un estrato herbáceo con cobertura muy importante de gramíneas. Así, el espectro biológico de la formación mostró el predominio de terófitas y geófitas las cuales representan el principal componente de alimentación para los micromamíferos cuyos hábitos tróficos son mayoritariamente de tipo granívoro (cf. Fulk 1975).

Los micromamíferos ocupan principalmente el suelo, cuando existe refugio, dividiendo los microhábitats horizontalmente antes que verticalmente; algunas especies explotan los espacios abiertos entre arbustos y otras el suelo existente debajo o cerca de la vegetación (Muñoz- 
Gayana 74(1), 2010

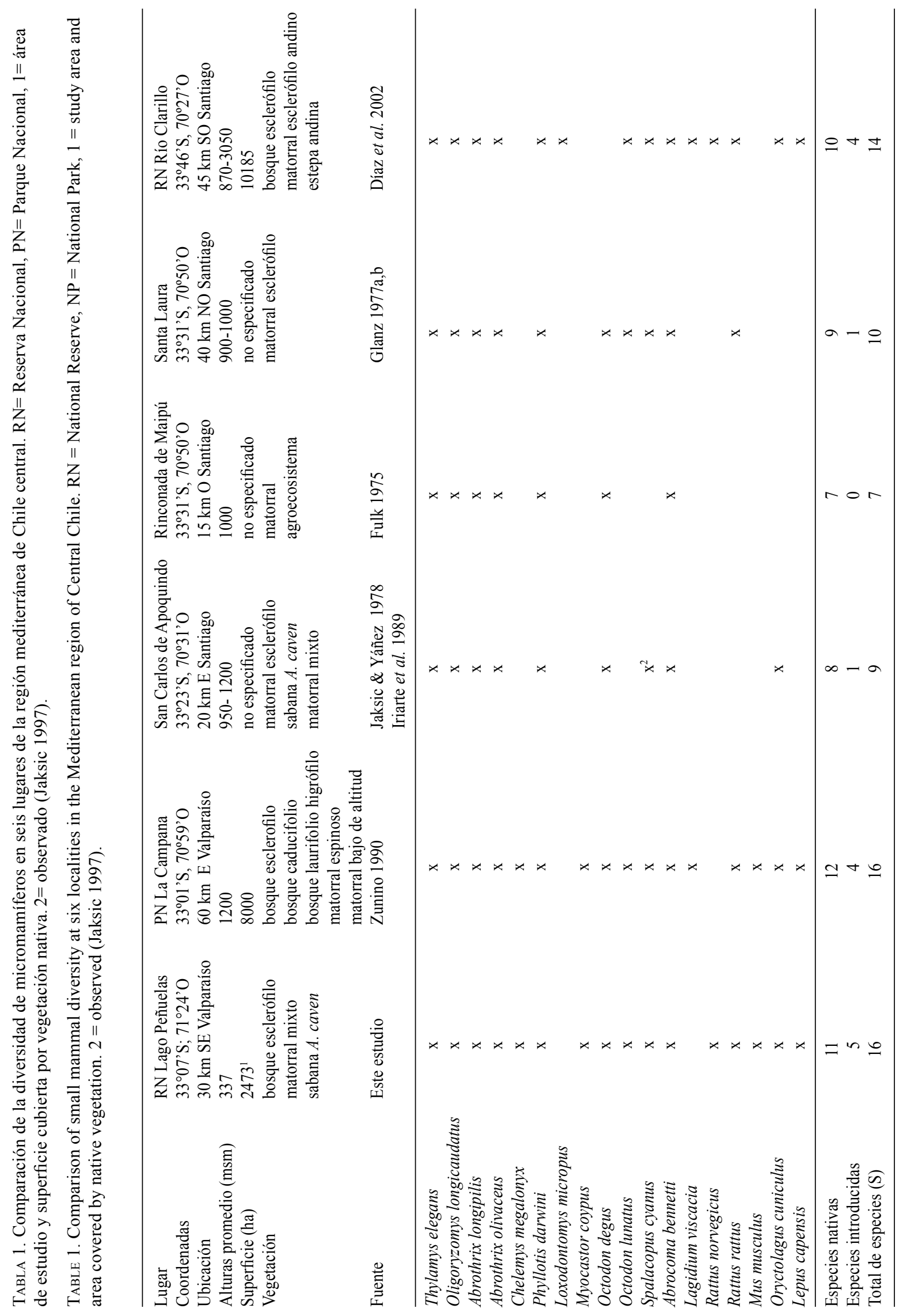


Pedreros \& Murúa 1989). Así, la menor diversidad de micromamíferos en la sabana de $A$. caven puede explicarse por las dimensiones tróficas (poco alimento) y espaciales (baja heterogeneidad espacial). Gallardo-Santis et al. (2005) proponen que también puede contribuir la capacidad de usar los hábitat verticales y documentan que la supuesta baja capacidad de escalar vegetación de los micromamíferos es relativa, ya que es alta en $T$. elegans y dependiente del diámetro del árbol en $P$. darwini.

De este modo la relación directa entre estructura (e.g., complejidad) de la vegetación y la diversidad de mamíferos es bien documentada (e.g., Malcolm 1997, Cruz-Lara et al. 2004); así, la mayor diversidad de micromamíferos del bosque esclerófilo podría explicarse por la mayor heterogeneidad espacial y productividad de dicho hábitat, factores complementarios cuyo modo de acción es la gama de recursos disponibles y la amplitud media del nicho (Pianka 1982, August 1983). En términos generales, ambientes estructuralmente más complejos ofrecen más variedad de microhábitats distintos que aquellos ambientes más simples. Por otro lado, los ambientes más productivos, o los ambientes donde el alimento es abundante, ofrecen más elección de presas $\mathrm{y}$, por tanto, permiten mayor especialización dietaria que los ambientes menos productivos. Considerando estos aspectos, se explicaría que la sabana de $A$. caven, por ser un ambiente estructuralmente simple y con una oferta alimentaria homogénea, registre una diversidad de especies menor comparada con la encontrada en otros ambientes. Esta relevancia de la heterogeneidad espacial y la productividad es concordante con lo documentado por Muñoz-Pedreros (1992) en ensambles de micromamíferos en matorrales esclerófilos degradados, en una matriz de plantaciones de $P$. radiata en la provincia de Concepción, asentados sobre territorios originalmente cubiertos con bosques esclerófilos.

Las formaciones vegetacionales del tipo bosque esclerófilo y matorral mixto presentes en la zona central de Chile, especialmente las presentes en la RNLP, conforman un importante núcleo de diversidad faunística. La diversidad de micromamíferos de la RNLP es concordante con los ecosistemas que representa como área silvestre protegida (e.g., bosque esclerófilo, sabana de $A$. caven), siendo la diversidad $\alpha$ del bosque esclerófilo y del matorral mixto más determinantes que la diversidad $\beta$.

La transformación de bosques y matorrales es el principal factor de cambio de uso del suelo en Chile, provocando la pérdida de hábitat con la consiguiente pérdida de biodiversidad (Arroyo 1999, Echeverría et al. 2006, Aguayo et al. 2009). Sólo un 2,0\% (ca. 6835 ha) del total de bosque esclerófilo típico de la ecorregión del matorral que aún existe (sobre 345000 ha) se encuentra protegido por el Sistema Nacional de Areas Silvestres Protegidas por el Estado (Díaz et al. 2002). Si bien es cierto que la RNLP conserva bien la diversidad de micromamíferos en el bosque esclerófilo y en el matorral mixto, el evidente grado de deterioro de estos ambientes ponen en riesgo esta diversidad a escala de paisaje (véase Muñoz et al. 1996, Smith-Ramírez et al. 2005). Considérese que del total de la superficie de la RNLP sólo el 21,1\% mantiene la cubierta vegetal nativa mientras el resto de la reserva lo constituyen plantaciones forestales exóticas. De esta forma, es necesario promover la conservación de áreas de bosque esclerófilo suficientemente amplias y continuas, ya sea en otras áreas protegidas o en áreas privadas, de manera de asegurar la viabilidad de este tipo vegetacional y de su fauna asociada. Se podrían explorar opciones de restauración ecológica y establecimiento de conectividad (e.g., corredores biológicos en la red hídrica) para facilitar el movimiento de la fauna que vive en los fragmentos de bosque esclerófilo de la zona central de Chile.

\section{AGRADECIMIENTOS}

A la Corporación Nacional Forestal (CONAF) Región de Valparaíso y al Centro de Estudios Agrarios y Ambientales (CEA). También a Javiera Meza y Santiago Huaiquinao (CONAF) y Patricia Möller (CEA). A Guillermo D’Elía y dos revisores anónimos que contribuyeron poderosamente con sus críticas, observaciones y sugerencias.

\section{BIBLIOGRAFÍA}

Arroyo, M.T.K. 1999. Criterios e indicadores para la conservación de la biota de los ecosistemas mediterráneos. Revista Chilena de Historia Natural 72(4): 473-474.

Aguayo, M., A. Pauchard, G. Azócar \& O. Parra. 2009. Cambio del uso del suelo en el centro sur de Chile a fines del siglo $\mathrm{XX}$ : entendiendo la dinámica espacial y temporal del paisaje. Revista Chilena de Historia Natural 82(3):361-374.

August, P. 1983. The rol of habitat complexity and heterogeneity in structuring tropical mammal communities. Ecology 64:1495-1507.

BraY, J.R. \& J.T. Curtis. 1957. An ordination of the upland forest communities of southern Wisconsin. Ecological Monograph 27:325-349.

Cabrera, A.L. \& A. Willink. 1973. Biogeografía de América Latina. Serie de Biología, Monografía $N^{\circ} 13$, Programa Regional de Desarrollo Científico y Tecnológico, Departamento de Asuntos Científicos, Secretaría General de la Organización de los Estados Americanos, Washington D.C. 120 pp.

Ceballos, G. \& P.R. Ehrlich. 2006. Global mammal distributions, biodiversity hotspots, and conservation. Proceedings of the National Academy of Sciences 103:19374-19379. 
Cornell, H.V. \& J.H. Lawton. 1992. Species interactions, local and regional processes, and limits to the richness of ecological communities: a theoretical perspective. Journal of Animal Ecology 61:1-12.

Cruz-Lara, L., C. Lorenzo, L. Soto, E. Naranjo \& N. RamírezMarcial. 2004. Diversidad de mamíferos en cafetales y selva mediana de las cañadas de la selva Lancadona, Chiapas, México. Acta Zoológica Mexicana 20(1):63-81.

DeBlase, A.F. \& R.E. Martin. 1974. A manual of mammalogy. Wm, C. Brown Company Publishers, Dubuque, Iowa, USA. 329 pp.

Di CASTRI, F. 1973. Climatographical comparison between Chile and the western coast of North America. In: Mediterranean-type ecosystems: origins and structure (Eds. di Castri, F. \& Mooney, H.A.), pp. 21-36. SpringerVerlag, Berlin.

Di Castri, F. \& E.R.Hajek. 1976. Bioclimatología de Chile. Ediciones Universidad Católica de Chile, Santiago de Chile. 128 pp.

Díaz, I., C. Sarmiento, L. Ulloa, R. Moreira, R. Navia, E. Véliz \& C. PeÑa. 2002. Vertebrados terrestres de la Reserva Nacional Río Clarillo, Chile Central: representatividad y conservación. Revista Chilena de Historia Natural 75(2):433-448.

Dinerstein, E., D.M. Olson, A.L. Webster, S.A. Primm, M.P. BROOKBINDER \& G. LEDEC. 1995. A Conservation Assessment of the Terrestrial Ecoregions of Latin America and the Caribbean. The World Bank, Washington, D. C. 152 pp.

Echeverría, C., D. Coomes, J. Salas, J.M. Rey-Benayas, A. Lara \& A. Newton. 2006. Rapid deforestation and fragmentation of Chilean Temperate Forests. Biological Conservation 130(4): 481-494.

Ellemberg, H. \& D. Mueller-Dombois. 1966. A key to Raunkiaer plant life forms with revised subdivision. Berichte des Geobotanischen Institutes, ETH Stiftung Rübel 37:56-73.

FulK, W. 1975. Population ecology of rodents in the semiarid shrublands of Chile. Occasional Papers Museum Texas Tech University 33:1-40.

Gajardo, R. 1994. La vegetación natural de Chile. Clasificación y distribución. Editorial Universitaria, Santiago. 165 pp.

Gallardo-Santis, A., J. Simonetti \& R. VÁsquez. 2005. Influence of tree diameter on climbing ability of small mammals. Journal of Mammalogy 86(5):969-973.

Gaston, K.J. \& T.M. Blackburn. 2000. Pattern and process in macroecology. Blackwell Science, Oxford. 377 pp.

Glanz, W. 1977a. Small mammals. In: Chile-California Mediterranean scrub atlas: a comparative analysis (Eds. Thrower, N.J. \& Bradbury D.E.), pp 232-237. Dowden Hutchinson \& Ross. Stroudsburg, Pennsylvania.

Glanz, W. 1977b. Comparative ecology of small mammal communities in California and Chile. Ph.D. dissertation, University of California, Berkeley.

Greuter, W. 1995. Extinctions in Mediterranean areas. In: Extinction rates (Eds. Lawton, J.H. \& May, R.M.), pp. 88-97. Oxford University Press, New York. 248 pp.

Grigera, D., C. Brion, J.O. Chiapella \& M.S. Pillado. 1996. Las formas de vida de las plantas como indicadores de factores ambientales. Medio Ambiente 13:11-29.

Hauenstein, E., C. Ramírez, M. Latsague \& D. Contreras. 1988. Origen fitogeográfico y espectro biológico como medida del grado de intervención antrópica en comunidades vegetales. Medio Ambiente 9(1):140-142.

Hauenstein, E., A. Muñoz-Pedreros, J. Yáñez, P. SÁnchez, P. Möller, B. GuíÑEz \& C. Gil. 2009. Flora y vegetación de la Reserva Nacional Lago Peñuelas, Reserva de la Biosfera Región de Valparaíso, Chile. Bosque 30(3):159179.

Iriarte, J.A., L.C. Contreras \& F.M. Jaksic. 1989. A long-term study of a small-mammal assemblage in the central Chilean matorral. Journal of Mammalogy 70: 79-87.

JAKSIC, F. 1997. Ecología de los vertebrados de Chile. Ediciones Universidad Católica de Chile. 261 pp.

JAKSIC, F. \& J. YÁÑEZ. 1978. Variación anual de la composición comunitaria de micromamíferos en Los Dominicos, Santiago. Noticiario Mensual del Museo Nacional de Historia Natural (Chile) 271:10-11.

Köppen, W. 1948. Climatología. Editorial Fondo de Cultura Económica. México. 158 pp.

Krebs, C. 1985. Ecología. Estudio de la distribución y la abundancia. $2^{\text {a }}$ Edición. Editorial Interamericana. 753 pp.

MagurRan, A.E. 1998. Ecological Diversity and its Measurement. Princeton University Press, Princeton, New Yersey. 179 pp.

Malcolm, J. 1997. Biomasa and diversity of small mammals in Amazonian Forest fragmented communities. In: Tropical Forest Remnants: Ecology, Management, and Conservation of Fragmented Communities (Eds. Laurence, W.F. \& Bierregaard, R.O. Jr.), pp. 207-221. The University of Chicago Press. Chicago. USA. 616 pp.

Maldonado, S. 1986. La dieta de la lechuza (Tyto alba) en la Reserva Forestal Lago Peñuelas. Departamento Técnico V Región. Corporación Nacional Forestal, Ministerio de Agricultura. 14 pp.

ManN, G. 1978. Los pequeños mamíferos de Chile. Gayana (Zoología) 40:1-341.

Mardones, G. 1996. Representatividad biogeográfica del Sistema Nacional de Áreas Silvestres Protegidas del Estado de Chile. Tesis Escuela de Geografía, Universidad Católica de Chile.

Marticorena, C. \& M. Quezada. 1985. Catálogo de la flora vascular de Chile. Gayana Botánica 42(1-2):5-157.

Matthei, O. 1995. Manual de las malezas que crecen en Chile. Alfabeta Impresores, Santiago de Chile. 545 pp.

McAleece, N. 1998. Biodiversity: Professional Beta 1. The Natural History Museum and the Association for Marine Science. London, United Kingdom. http://www.sams.ac.uk.

Medail, F. \& P. Quezel. 1997. Hot-Spots Analysis for Conservation of Plant Biodiversity in the Mediterranean Basin. Annals of the Missouri Botanical Garden 84(1):112-127.

Mella, J. \& J. Simonetti. 1994. Representación de poblaciones viables: Conservación de mamíferos en las áreas silvestres protegidas de Chile. Ambiente y Desarrollo 10:72-78.

Moreno, C. 2001. Método para medir la biodiversidad. Centro de Investigaciones Biológicas, Universidad Autónoma del Estado de Hidalgo. México. 83 pp.

MuÑoz, M., H. NúÑEz \& J. YÁÑEZ. 1996. Libro Rojo de los sitios prioritarios para la conservación de la diversidad biológica en Chile. Ministerio de Agricultura, Corporación Nacional Forestal, Santiago de Chile. 203 pp.

Muñoz Pedreros, A. C. Gil, J. Yáñez, \& J. Rau. 2010. Raptor habitat management and its implication on the biological 
control of the Hantavirus. European Journal of Wildlife Research DOI 10.1007/s10344-010-0364-2.

Muñoz-Pedreros, A. 1992. Ecología del ensamble de micromamíferos en un agroecosistema forestal de Chile Central. Una comparación latitudinal. Revista Chilena de Historia Natural 65(4):417-428.

Muñoz-Pedreros, A. \& R. Murúa. 1989. Efectos de la reforestación con Pinus radiata sobre la diversidad y abundancia de los micromamíferos en un agroecosistema de Chile Central. Turrialba 39(2):143-150.

Muñoz-Pedreros, A. \& J. Rau. 2004. Estudio de egagrópilas en aves rapaces. En: Aves Rapaces de Chile (Eds. MuñozPedreros, A., Rau, J. \& Yáñez, J.), pp. 265-279. CEA Ediciones. 387 pp.

Muñoz-Pedreros, A. \& C. GIL. 2009. Orden Rodentia. En: Mamíferos de Chile (Eds. Muñoz-Pedreros, A. \& Yáñez, J.), pp. 93-157. Segunda Edición. CEA Ediciones. 573 pp.

Muñoz-Pedreros, A. \& J. YÁñez. (eds). 2009. Mamíferos de Chile. Segunda edición CEA Ediciones. 573 pp.

Muñoz-Pedreros, A., R. Murúa \& L. González. 1990. Nicho ecológico de micromamíferos en un agroecosistema forestal de Chile central. Revista Chilena de Historia Natural 63(3):267-277.

Myers, N., R.A. Mittermeier, C.G. Mittermeier, G. Da Fonseca \& J. KENT. 2000. Biodiversity hotspots for conservation priorities. Nature 403:853-858.

NARANJo, J. 1993. Situación de la conservación de la biodiversidad en el Sistema Nacional de Áreas Protegidas de Chile. Parque Nacional Iguazú, Argentina CONAF. 40 pp.

Oberdorfer, E. 1960. Planzensoziologishe Studien in Chile - Ein Vergleich mit Europa. Weinheim. Cramer. 208 pp.

Osgood, H. 1943. The mammals of Chile. Field Museum of Natural History, Zoological series 30:1-268.

Pearson, O. 1995. Annotated keys for identifying small mammals living in or near Nahuel Huapi National Park or Lanin National Park, southern Argentina. Mastozoología Neotropical 2:99-148.

Pianka, E. 1982. Ecología evolutiva. Universidad de Texas, Austin. Ediciones Omega, S.A., Barcelona. 365 pp.

Pine, R.H., S.D. Miller \& M.L. Schamberger. 1979. Contributions to the Chilean mammalogy. Mammalia 43:339-376.

Purvis, A. \& P. Raven. 2000. Extincion by numbers. Nature 403:843-845.

Quintana, V. 2009. Registros de Dromiciops gliroides y Chelemys megalonyx en bosques nativos del Centro Sur de Chile. Gestión Ambiental 17: 45-54.

Ramírez, C. 1988. Formas de vida, fitoclimas y formaciones vegetales. El Árbol...Nuestro Amigo 4:33-37.

ReISE, D. 1973. Clave para la determinación de los cráneos de marsupiales y roedores chilenos. Gayana: Zoología 27:1-20.

RickLEFs, R.E. \& D. SCHLUTER. 1993. Species diversity in ecological communities, historical and geographical perspectives. University of Chicago Press, Chicago. 414 pp.

Rodríguez, P., J. Soberón \& H.T. Arita. 2003. El componente beta de la diversidad de mamíferos de México. Acta Zoológica Mexicana 89:241-259.

Scott, J.M., E.A. Norse, H.T. Arita, A. Dobson, J.A. Estes, M. Foste, B. Gilbert, D. Jensen, R.L. Knight, D. Mattson \& M.E. SoulÉ. 1999. The issue of scale in selecting and designing biological reserves. In: Continental Conservation, scientific foundations of regional networks (Eds. Soulé, M.E. \& Terborgh, J.), pp, 19-38. Island Press, Washington D. C. 227 pp.

Simonetti, J. 1999. Diversity and conservation of terrestrial vertebrates in mediterranean Chile. Revista Chilena de Historia Natural 72:493-500.

Simonetti, J. \& A. Walkowiak. 1979. Presas de Tyto alba Gray, 1829 (Aves: Strigidae) en el Parque Nacional La Campana. Anales del Museo de Historia Natural de Valparaíso (Chile) 12:89-91.

Simonetti, J., T. Arroyo, E. Spotorno \& E. Lozada. 1995. Diversidad biológica de Chile. Comité Nacional de DiversidadBiológica, Comisión Nacional de Investigación Científica y Tecnológica. Santiago, Chile. 364 pp.

Smith-Ramírez, C., J.J. Armesto \& C. Valdovinos (eds). 2005. Biodiversidad y Ecología de los Bosques Costeros de Chile. Editorial Universitaria. 708 pp.

Tirira, D. 1999. Mamíferos del Ecuador. Publicación especial. $392 \mathrm{pp}$.

Whittaker, R.H. 1960. Vegetation in the Siskiyou Mountains, Oregon and California. Ecological Monographs 30:279338.

WhitTAKer, R.H. 1972. Evolution and measurement of species diversity. Taxon 21: 213-215.

Whittaker, R.J., K.J. Willis \& R. Field. 2001. Scale and species richness: towards a general, hierarchical theory of species diversity. Journal of Biogeography 28:453-470.

Wikum, D. \& G.F. Shanholtzer. 1978. Application of the BraunBlanquet cover - abundance scale for vegetation analysis in land development studies. Environmental Management 2(4):323-329.

YÁÑez, J. \& A. Muñoz-Pedreros. 2009. Mamíferos vivientes de Chile. En: Mamíferos de Chile (Eds. Muñoz-Pedreros, A. \& Yáñez, J.), pp. 47-50. Segunda Edición. CEA Ediciones. 573 pp.

ZAR, J. 1996. Biostatistical Analysis. Third Edition. Prentice Hall Inc, New Jersey. USA. 662 pp.

Zunino, S. 1990. El Parque Nacional La Campana y su fauna teriológica. Comunicaciones del Museo Regional de Concepción 4:23-29.

Zunino, S. \& M. ArCos. 1989. Nuevos antecedentes de la dieta de Tyto alba en el Parque Nacional La Campana. Anales del Museo de Historia Natural de Valparaíso (Chile) 20:99-101.

Recibido: 15.10 .08

Aceptado: 04.04.10 